\title{
Use of analogy in learning physics: The role of representations
}

\author{
Noah S. Podolefsky and Noah D. Finkelstein \\ Department of Physics, University of Colorado at Boulder, Boulder, Colorado 80309, USA
}

(Received 13 March 2006; published 18 July 2006)

\begin{abstract}
Previous studies have demonstrated that analogies can promote student learning in physics and can be productively taught to students to support their learning, under certain conditions. We build on these studies to explore the use of analogy by students in a large introductory college physics course. In the first large-scale study of its kind, we demonstrate that different analogies can lead to varied student reasoning. When different analogies were used to teach electromagnetic (EM) waves, we found that students explicitly mapped characteristics either of waves on strings or sound waves to EM waves, depending upon which analogy students were taught. We extend these results by investigating how students use analogies. Our findings suggest that representational format plays a key role in the use of analogy.
\end{abstract}

DOI: 10.1103/PhysRevSTPER.2.020101

PACS number(s): 01.40.Fk

\section{INTRODUCTION}

Analogies are ubiquitous in physics. They are used by working physicists, physics teachers, and students learning physics. In formulating a theory of electrical phenomena, James Clerk Maxwell claimed, "Instead of using the analogy of heat, a fluid, the properties of which are entirely at our disposal, is assumed as the vehicle of mathematical reasoning....The mathematical ideas obtained from the fluid are then applied to various parts of electrical science." 1

While Maxwell used analogies to generate a new physical theory, physicists also use analogies to communicate ideas to other scientists ${ }^{2,3}$ and to the public. For instance, Cooper pairs in a fermionic condensate are likened to pairs of "shy people circling each other in a crowded room." 4,5 Some analogies can serve both to communicate ideas and to generate new scientific knowledge. Consider Rutherford's planetary model of the atom. While the original utility was generative ${ }^{6}$ - producing a model that explained experimental results (which it accomplished better than competing analogies, such as the "plum pudding" model) — this analogy is often used to communicate an introductory atomic model to physics students. Thus, analogies are not only useful to working physicists, but to physics teachers as well. In this paper, we explore the role of analogy in teaching undergraduate physics. Our work builds upon previous experimental and theoretical studies of analogy in physics teaching and learning.

A prevailing view is that an analogy can be treated as a mapping from a base domain (e.g., the solar system) to a target domain (e.g., the atom). ${ }^{7-10}$ Applying this framework, experimentalists have asked specific research questions about the use of analogy in teaching physics. For example, Gentner explored different analogies for electric circuitswater in a pipe vs moving crowds-and found that different analogies led to different student reasoning about electric circuits. ${ }^{8}$ These results supported the hypothesis that analogies generate inferences, in this case about electric circuits. For example, using a water analogy, students mapped water reservoirs to batteries and were better prepared to answer questions about batteries than students who used a moving crowd analogy (which does not include an equally useful analog to batteries). To be sure, not all inferences are equally likely to map, nor do all mappings generate correct inferences. Furthermore, Gentner found that while electric circuit analogies that were produced by students were effective for generating inferences, attempts to teach the same analogies were not as effective. Other researchers, however, have formulated teaching methods using analogy, ${ }^{11}$ and it has been shown that teaching with analogy can be effective in physics. ${ }^{12,13}$

In summary, previous researchers have made claims that analogies are generative, and that teaching with analogy can sometimes be productive. Questions remain as to how analogies can be used in a teaching mode, and whether previous results can be replicated with a larger number of students $(N>200)$ in an authentic learning environment (i.e., an introductory physics course). We wish to test the hypothesis that analogies generate inferences with large $N$ in a college setting, to study how they affect student reasoning, and begin to delineate a mechanism by which analogies may be applied productively. In this study, we address these issues by focusing on analogies for teaching and learning electromagnetic (EM) waves.

Of the concepts taught in introductory physics, the EM wave is one of the most abstract. Students have difficulty interpreting graphical representations ${ }^{14}$ of EM waves and relating them to the physical phenomena. ${ }^{15}$ Unlike more concrete wave phenomena (e.g., oscillations of a string), EM waves are variations in fields-the fields represent potential forces on charges. Furthermore, students are taught that, for a plane wave, the fields exist everywhere in space and the wave propagates even in the absence of a medium. ${ }^{16}$ For these reasons, we believe that EM waves can provide a fruitful content area for studying analogy. In this study, we ground the abstract concept of EM waves with more concrete phenomena: sound waves or waves on a string.

\section{THEORETICAL FRAMES}

Drawing on the dominant prior theoretical frames of students' use of analogy, we designed a study to examine whether, how, and when students use analogies. To introduce the theoretical frames, let us return to Rutherford's solar sys- 
tem analogy discussed earlier. Existing theory defines a structure for this analogy. Gentner ${ }^{7}$ has proposed that an analogy can be treated as a mathematical isomorphism. Symbolically, an analogy is the mapping $M: B \rightarrow T$, where $B$ is the base domain (e.g., the solar system) and $T$ is the target domain (e.g., the atom). Gentner calls this theoretical framework structure mapping. To use the analogy is to complete a mapping from one structure to another. Applying this theory to Rutherford's analogy, the sun maps to the nucleus, the planets to the electrons, the gravitational force to the Coulomb force, etc. In addition, relations between these objects, such as "revolves around," map across domains. This analogy is commonly employed to teach a particular, though rudimentary, model of the atom to students. This model has certain features that are useful for understanding how atoms work: the nucleus is at the center with electrons orbiting, held in by some central force; electrons are tiny compared to the nucleus, and most of the atom is empty space. This model lays in contrast to other analogies, such as the "plum pudding" or "electron cloud" models, which ascribed very different characteristics to atoms.

Structure mapping belongs to a class of theories that might be categorized as abstract transfer. ${ }^{12}$ Applying a structure mapping theory to instruction rests on three assumptions: First, analogies are assumed to be inherently linearmappings are made directly from one structure to another. A second assumption is that students possess a mostly complete understanding of the base domain and little or no understanding of the target domain. The third assumption is that students will accept that the analogy relation is valid and be able to complete the mapping correctly. Rather than focus on students' ability to use an analogy, abstract transfer theories tend to rate an analogy's effectiveness based on the robustness of the analogy, the structure of which has been defined $a$ priori. ${ }^{17}$ In other words, analogies are framed from the expert physicist's point of view, not the student's. While these models are useful for framing our understanding of analogies, they fall short of explaining how analogies are used by students, or how to use analogies productively for teaching.

A response to the abstract transfer approach to analogy is that of "bridging." 12,13 A bridging analogy provides intermediate steps to help students make sense of an analogy. Rather than assume students have little or no understanding of the target domain, bridging strategies assume that students have some conceptions about the target, albeit students' ideas may be in error. The bridging analogy is intended to promote conceptual change. For example, consider a teacher who is trying to explain Newton's third law. The teacher puts a book on a table and asks the student if the table exerts a force on the book. A common student response is that the table does not exert a force-it simply gets in the way to prevent the book from falling. As a bridging analogy, the teacher proposes that the book is resting on a spring instead. The student accepts that the spring exerts a force on the book, but does not yet accept that the table exerts a force (maintaining that the table gets in the way). The "bridge" that the teacher suggests is that the table itself bends under the weight of the book - the table is "springy." The bridge provides a way for the student to grasp that the table does exert a force back on the book. This approach to analogy use appears to differ from Gentner in a subtle way. Rather than framing the analogy in terms of a direct mapping, a number of intermediate mappings are employed that build on students' prior conceptions. The sequence of mappings is not necessarily linear, but often forms a richly interconnected network with many bases and targets connected in multiple ways to others. The end result of such a sequence is that, in the case described above, the teacher attempts to combine the features of two domains-tables and springs. Brown and Clement refer to this process as enriching the target domain. An alternative perspective is that domains mix into a conceptual blend. ${ }^{18,19}$ The springy table blend is an abstract mental construct created by blending the objects table and spring. We claim that structure mapping, bridging analogies, and blending are mutually supportive theoretical frames. Structure mapping provides a useful way of describing analogies in terms of mapping, and allows instructors to identify mappings in the analogy to be taught. Blending describes a cognitive process of combining domains, and bridging analogies describe a particular way students construct and then use blends to learn new ideas.

Expert physicists commonly use analogies productively, even when solving basic physics problems. ${ }^{20}$ In fact, physicists seem to be experts at using analogies: they know when an analogy generates correct inferences, and recognize when it fails. Students, unfamiliar with the content to be learned, are not necessarily able to make such productive use of analogies, especially when using analogies to learn about concepts that are very abstract or unfamiliar. ${ }^{12}$ Structure mapping delineates what is means for an analogy to "work," and what it means for an analogy to "break down" in terms of mapping. For example, in the planetary model of the atom, the attribute "massive" should map from the sun to the nucleus, but not the attribute "yellow." The relation "sun attracts planet" maps to "nucleus attracts electron," but "planet attracts planet" does not map to electrons. Structure mapping predicts that attributes and relations are more likely to map if they are tightly integrated into a hierarchy of connected ideas. For instance, structure mapping predicts that "massive" will map because it is a key component of a higher order structure, i.e. a central force system. Yellow is not as likely to map because it is not a necessary component of a central force system. In this study, we use structure mapping to identify analogical mappings, but not as a mechanism by which students use analogies in their learning. Brown and Clement ${ }^{12}$ distinguish between expert and novice usage of analogies, and address the challenge of novice usage specifically with a bridging strategy that builds on students' prior conceptions. We build on the ideas of mapping between domains, layering of more complex or abstract ideas, and blending domains to examine how and when students successfully use analogies.

\section{RESEARCH QUESTIONS}

In this paper we address the following questions. Does the use of different analogies lead to different student reasoning in a large physics class at the college level, and, further, do 
analogies support the generation of inferences when taught in this environment? These questions lead us to examine some of the key mechanisms by which students productively use analogies. Our findings are summarized as follows: Analogies can lead students to generate ideas (i.e., they are generative). Further, these analogies can be taught. We demonstrate these results in a large-scale study focusing on undergraduate physics. Furthermore, we begin to examine key mechanisms by which students use analogies, and find that representations are crucial to student reasoning and the promotion of certain analogical mappings.

\section{COURSE DESCRIPTION}

Our experiment was carried out in two introductory physics courses at a large university. Both courses were calculusbased introductory physics, one taught in spring 2005 ( $N$ $=249)$ and one in fall $2005(N=353)$. Both were the second semester of a two semester sequence, primarily covering electricity and magnetism. Each course consisted of three one-hour lectures per week, in a conventional lecture hall, and one hour in a small recitation setting $(N \sim 25)$ led by two teaching assistants. The lectures made extensive use of Peer-Instruction ${ }^{21}$ and personal electronic response systems. ${ }^{22}$ Otherwise, lectures were the traditional style, with the instructor lecturing from the front of the room with chalk, overheads, and an occasional demonstration. During the recitations, students worked in small groups using the Tutorials in Introductory Physics. ${ }^{23}$ Students generally completed a tutorial pretest online, submitted before the start of recitation.

The initial run of our experiment (spring) was designed as a preliminary study. We modified the follow-up study (fall) based on results of the first in three key ways. (1) The sole focus of the recitation was on paper and pencil based tutorials. The first run included other activities. (2) Based on the results of the initial study, we refined the tutorial used in the follow-up study. (3) We more tightly coupled the post-test to the treatment by administering the test sooner after the treatment, and by placing the experiment so no other relevant instruction other than the tutorial occurred before the test. The follow-up study was meant to demonstrate that our initial findings were repeatable, and that our approach to teaching with analogies could be refined. Our studies were conducted in two parts. Part I examined the generative use of analogies. Part II examined mechanisms behind the use of analogy, focusing on representations.

\section{PART I: TEACHING WITH ANALOGIES}

\section{Description of teaching materials}

In the first portion of our study, students learned about EM waves from a tutorial which borrowed heavily from the Tutorials in Introductory Physics. Students completed the tutorial in recitation sections. We modified the original tutorial to include a front section that focused on analogies. In each course, students were randomly assigned by recitation to one of three groups, denoted as the string analogy, sound analogy, and no-analogy groups. All the students in a given recitation section were placed into the same group, and recita-
TABLE I. Two treatment groups (string and sound) and control group (no analogy) for the initial (spring) and follow-up (fall) studies.

\begin{tabular}{lll}
\hline \hline Course & \multicolumn{1}{c}{ Group } & N \\
\hline \multirow{3}{*}{ Spring 2005 } & String Analogy & 72 \\
& No analogy & 90 \\
& Sound analogy & 87 \\
\hline \multirow{3}{*}{ Fall 2005 } & String analogy & 91 \\
& No analogy & 112 \\
& Sound analogy & 95 \\
\hline \hline
\end{tabular}

tions were evenly distributed among different teaching assistants and times of the day. Table I lists the numbers of students in each group for each course. Each group completed the modified EM waves tutorial, which consisted of three parts. For the analogy groups, part 1 covered basic wave concepts, such as amplitude, wavelength, and frequency, in the context of either sound waves or waves on a string. Part 1 for the no-analogy group was isomorphic to the analogy groups, but used EM waves instead of one of the analogies. Part 2 was substantially identical for all three groups and covered basic wave concepts for EM waves. Part 2 also used more sophisticated representations than part 1 , described in more detail below. Part 3 was unmodified from the original version from the Tutorials, covering concepts related to forces on charges from electric and magnetic fields. The tutorials for the three groups differed only in the use of the analogies, and were made as isomorphic as possible. In both courses, this tutorial provided students their first formal instruction on the content of the analogies used (string and sound waves).

In addition to covering the basic wave concepts listed above, the tutorials in the follow-up study (fall) were tailored to address specific concepts about the propagation of waves through space. In part 1, students were presented with one of the pictures shown in Fig. 1. The string and sound analogy groups were presented with the string and sound pictures, respectively, and the no-analogy group with the EM wave picture. In the string group, three beads are labeled 1-3. Students were asked to describe the motion of each bead as the wave propagates to the right. The intention of this exercise was to cue on two features of waves on strings: these are traveling waves (bead 2 moves) and two-dimensional (2D oscillations are confined to a vertical plane and bead 3 does not move). In the sound group, students were asked to describe the pressure at points $1-3$. Here, the intention was to cue on the $3 \mathrm{D}$ nature of sound waves (sound propagates as spherical wave fronts, and, hence, the pressure is nearly equal at all three points, very nearly like a plane wave). In the no-analogy group, students were asked to describe the magnitude of the electric field at points 1-3. Here, the intention was to cue on the 3D nature of EM waves (since this EM wave is a plane wave, the field is equal at all three points). Note that both the traveling and 3D wave characteristics are critical for understanding EM waves.

Although each picture in Fig. 1 uses a sinusoid to represent the wave, the sinusoid carries a different meaning for 

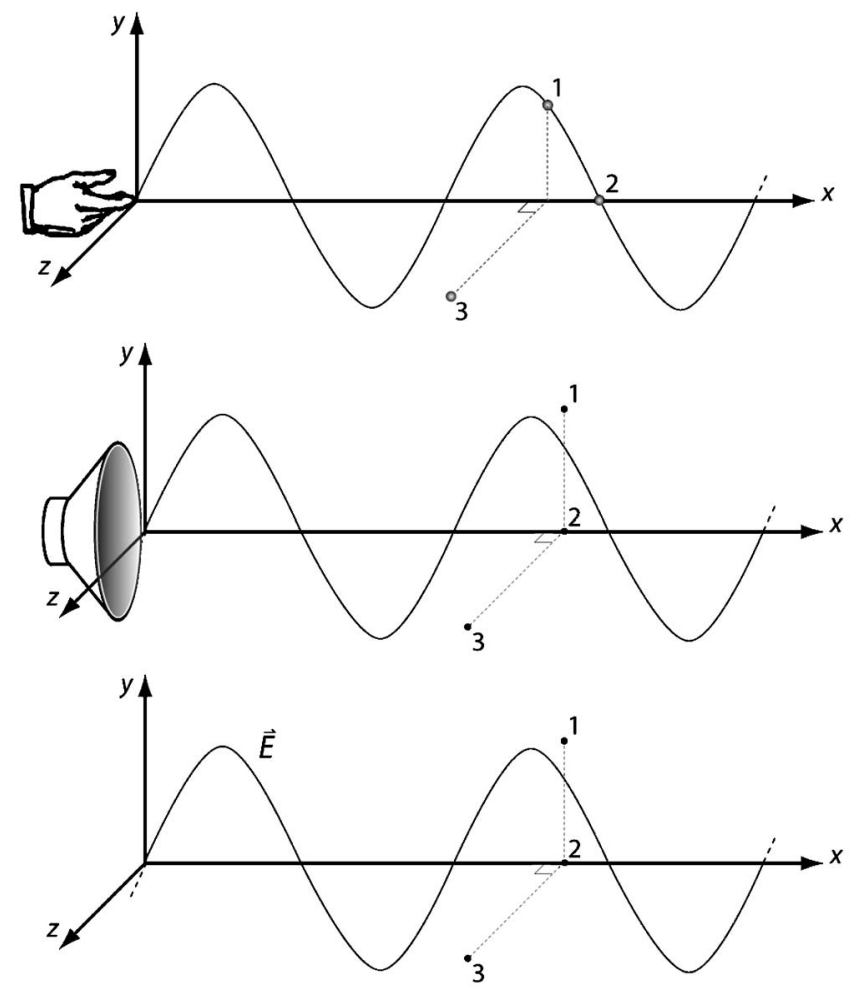

FIG. 1. String (top), sound (middle), and EM (bottom) wave pictures from part 1 of the tutorial. These were added to the tutorial in the follow-up (fall) study.

each. Specifically, the sinusoid for the string represents a string oscillating up and down-a transverse wave constrained to the $x-y$ plane. For the sound wave, however, the sinusoid represents the sound pressure (or motion of air particles) - a longitudinal wave propagating throughout space in three dimensions. Finally, for the EM wave, the sinusoid represents the magnitude of the electric and/or magnetic fields-a transverse wave propagating throughout space in three dimensions. Referring to Fig. 1, students were explicitly taught about traveling waves in the context of strings, and about 3D waves in the context of sound.

In part II, for both the initial and follow-up studies, students in all three groups were presented with the picture shown in Fig. 2, drawn from the Tutorials, and told that it represented an EM wave at one instant in time. Students were asked to rank the magnitude of the electric and magnetic fields at each point (1-4) in the image. Note that this

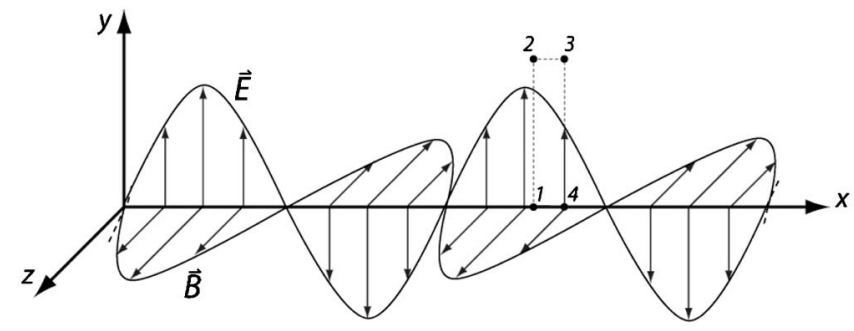

FIG. 2. EM wave picture from part 2 of the tutorial. This picture was used in both the initial and follow-up studies.

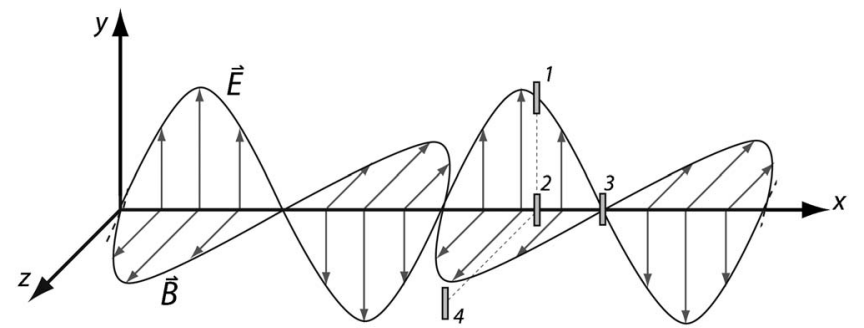

FIG. 3. EM wave as presented on the post-test in both the initial and follow-up studies. Vertical antennas are labeled 1-4.

exercise addresses the behavior of the electric and magnetic fields at four points in the $x-y$ plane, but not at other $z$ positions. Students in the two analogy groups were given a hint to use an analogy to sound or wave on a string in answering this question. However, the tutorials did not instruct students about which mappings to make (e.g., that an EM wave is like a sound in that it is $3 \mathrm{D})$.

\section{Post-test}

In each study, we assessed the effects of teaching with different analogies by giving a post-test which included a question that drew directly on the concepts covered in the tutorials. In the initial study, we gave an EM wave question on the final exam, given five weeks after the EM wave tutorial. The exam question presented students with a representation of an EM wave (Fig. 3) and explained that it showed a plane wave propagating to the right. The multiple choice question asked students to rank the time-averaged signals received by each antenna (labeled 1-4). In the follow-up study, we posed the same EM wave question as a concept test in the lecture on the day following the recitation in which students completed the EM wave tutorial. The concept test was given at the beginning of lecture, and students were instructed not to discuss the question before answering. Thus, for students in the follow-up study, the tutorial provided the only formal instruction prior to the post-test.

We anticipated particular outcomes from student responses to the post-test questions. While many studies examine only whether students answer questions correctly or incorrectly, here we attend to the information contained in which wrong answers (distracters) students choose..$^{24,25}$ In the analysis of post-test data, we look for effects of different analogies by examining student responses across different treatment groups (sound, string, or no analogy). We do not focus specifically on whether students chose the correct answer, but rather which treatment (analogy) preferentially leads to which response. We identify the characteristics "traveling" and "2D" as stringlike, and the characteristic "3D" as soundlike. ${ }^{26}$ Notably, the EM wave includes properties of both string and sound waves. We hypothesized that students in the string group would be more likely to choose stringlike distracters, while the sound group would be more likely to choose soundlike distracters. To be clear, this does not mean that we anticipated more students in the string group, for example, to choose the stringlike distracter over other distracters. Rather, we anticipated that students who 


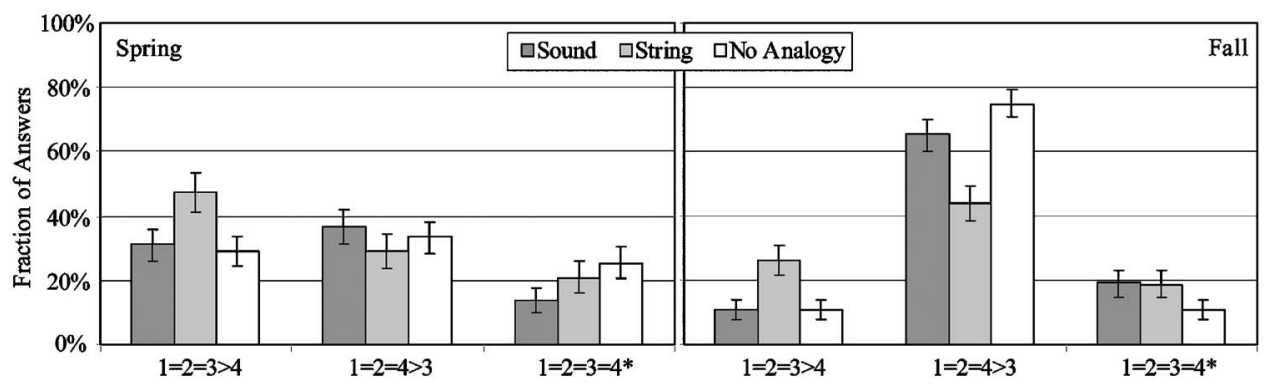

FIG. 4. Post-test results for the initial study (spring, left) and follow-up study (fall, right). The top three answers $(1=2=3>4,1=2$ $=4>3,1=2=3=4$ ) are shown below the horizontal axis. The analogy groups (sound, string, no-analogy) are indicated in the legend. Error bars are the standard error $(\sigma / \sqrt{n})$. The $*$ indicates the correct answer.

chose the stringlike distracter would be more likely drawn from the string group than the other groups. ${ }^{27}$ There may be many reasons why students answer in a particular way, and one distracter may prove stronger overall because of these reasons. Nonetheless, finding differences between treatment groups of the kind we have described would support the hypothesis that analogies generate inferences.

Of the five possible student responses, three dominated in both studies ( $>82 \%$ in spring, $>90 \%$ in fall), and no other distracter received a substantial fraction of responses $(<11 \%$ in spring, $<5 \%$ in fall). ${ }^{28}$ The top three responses are shown below the horizontal axis in Fig. 4 (from left to right: $1=2$ $=3>4,1=2=4>3$, and $1=2=3=4)$. We consider the first distracter $(1=2=3>4)$ to be associated with stringlike properties, and the second distracter $(1=2=4>3)$ to be associated with soundlike properties (as defined above). The third answer $(1=2=3=4)$ is correct and includes both string-like and soundlike properties.

The results of the post-test in the initial study are shown on the left side of Fig. 4. Overall, only $20 \%$ of students chose the correct answer $(1=2=3=4)$. Students from the string group were most likely to choose $1=2=3>4(p<0.05) .{ }^{29}$ There was no significant difference $(p>0.1)$ between the sound and no-analogy groups on the distracter $1=2=3>4$, nor were there significant differences $(p>0.1)$ between any of the groups on the distracter $1=2=4>3$. Students in the no-analogy group were slightly more likely to answer correctly compared to the sound group $(p=0.05)$. Notably, the post-test in the initial study assessed the effectiveness of a single recitation conducted five weeks earlier.

We found clear evidence that the different analogies affected student responses in the follow-up study. The results are shown on the right side of Fig. 4. Students from the string group were more likely than sound to choose $1=2$ $=3>4(p<0.01)$, while students from the sound group were more likely than string to choose $1=2=4>3 \quad(p<0.01)$. There were no significant differences $(p>0.1)$ between the sound and no-analogy groups on either distracter, nor were there significant differences between any of the groups on the correct answer $(1=2=3=4)$.

In summary, in both courses, students who chose the stringlike distracter were most likely from the string group, while students who chose the soundlike distracter were more likely from the sound and no-analogy groups. The soundlike distracter describes a wave extending throughout space in three dimensions, which is characteristic of both sound and EM waves. ${ }^{30}$ Notably, students in the no-analogy group were explicitly taught the 3D characteristic of EM waves. However, students in the sound group were only taught this characteristic of sound waves; these students were never explicitly taught this characteristic of EM waves. Thus, we believe students in the sound group were mapping this characteristic from sound to EM waves.

\section{PART II: REPRESENTATION AND ANALOGY}

In early student interviews, we found that representations could cue students to focus on different characteristics of EM waves. In order to study one possible mechanism for using analogies, we developed an assessment meant to probe student understanding of wave representations and associated phenomena. The assessment, described below, was given online, consisting of multiple choice and long answer questions. Students completed the assessment prior to recitation.

\section{Representation assessment}

In both studies, fall and spring, all students were divided evenly into two groups, denoted by string representation and sound representation groups. Table II shows the numbers of students in each group for each course. The representation assessment presented students with a pictorial representation of either a hand moving a string or a speaker and dust particle, shown on the left side of Fig. 5. Students were asked to choose the representation of the sound or string wave that made the most sense to them and then were asked to explain their choice as a long answer. The choices students selected from are the iconographic representations shown on the right side in Fig. 5. Students were told that there was no correct

TABLE II. Two groups for the wave representation assessment (string and sound) for the initial (spring) and follow-up (fall) studies.

\begin{tabular}{lll}
\hline \hline Course & \multicolumn{1}{c}{ Group } & $N$ \\
\hline Spring 2005 & String representation & 122 \\
& Sound representation & 122 \\
\hline Fall 2005 & String representation & 170 \\
& Sound representation & 168 \\
\hline \hline
\end{tabular}



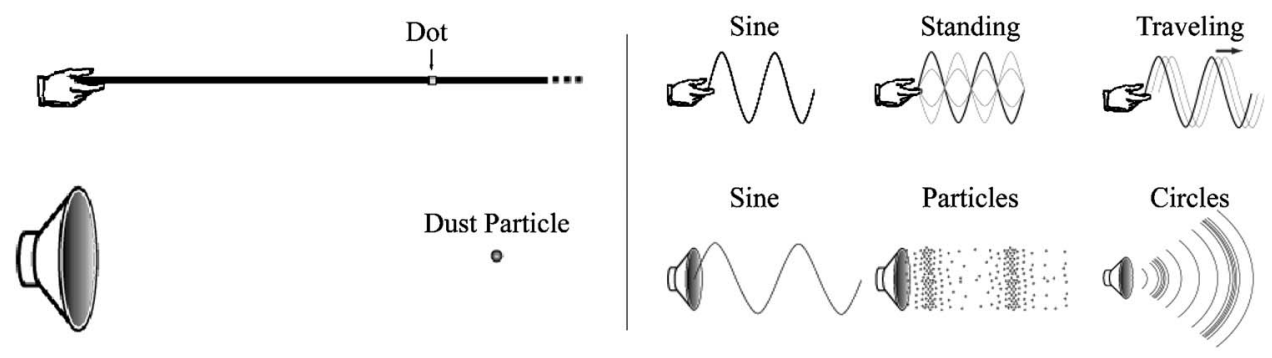

FIG. 5. Left: Pictorial representations from the string (top) and sound (bottom) wave representation assessments. Right: Iconographic representations from the wave representation assessments for strings (top) and sound (bottom). The representations are labeled only for reference in this paper-the labels did not appear in the representation assessment.

answer to the choice of representation. Students were then asked a multiple-choice follow-up question about the motion of either a dot on the string or the dust particle. The top three responses ${ }^{31}$ to the motion question were "up and down," "to the right," and "side-to-side."

To analyze the results of the representation assessment, we first examine the reasons students provided for choosing a particular iconographic representation. The reasons given were binned into categories based on an emergent coding scheme, described in more detail below. We next examined the relationship between which iconographic representations students choose and their answer on the motion question. Again, we look for information in the distracters as well as the correct answers. While students who choose different representations do not actually constitute different treatment groups, we look for associations between the choice of representation and the answer selected on the motion question.

Three dominant categories emerged from coding the reasons students gave for choosing a particular representation of sound. The categories are shown in Table III along with sample statements from students. "Formalism" is characterized by reference to mathematical objects associated with wave physics (e.g., usage of the word "sinusoidal"). "Medium" is characterized by reference to the medium through which the wave propagates (e.g., reference to "air particles"). "Spreads" is characterized by reference to sound as spreading, or traveling in multiple directions, as it propagates (e.g., in circles away from the speaker). Table IV presents the number of statements in each reasoning category sorted by selected iconographic representation. Approximately $15 \%$ of statements contained elements from two categories, and these statements were counted in each of these two categories. To test for the reliability of the coding scheme, two individuals (the lead author and a researcher unrelated to the study) coded a subset of the students' statements. Coding agreed to better than $87 \%$, and the patterns shown in Table IV were extremely consistent for the lead author's and other researcher's coding. There is a statistically significant relationship between students' choice of representation and reasoning given $\left(\chi^{2} ; p<0.001\right)$. The bulk of responses fall along the diagonal, suggesting association between representation and reason stated. Nearly all students who chose the sine or particle representation fell into the formalism or medium category, respectively. Students who chose the circles representation fell dominantly into spreads, with additional statements falling into medium. This result is similar for both semesters.
Four dominant categories emerged from coding the reasons students gave for selecting representations of the wave on a string. The categories are shown in Table $\mathrm{V}$ along with sample statements from students. "Formalism" is defined in the same way as the category for sound. "Transverse" is characterized by reference specifically to up/down motion of the string. "Traveling" is characterized by reference specifically to propagation along the length of the string. "Generic motion" is characterized by reference to motion of the string with no specific direction. Table VI presents the number of statements in each reasoning category sorted by selected iconographic representation. Again, approximately $15 \%$ of statements were counted in two different categories. Agreement between two separate coders was better than $82 \%$ in this instance. By examining the rows of Table VI, we find that each iconographic representation is associated with a different set of reasons. This relationship is statistically significant $\left(\chi^{2} ; p<0.01\right)$. However, the data in Table VI, for the string group, are more distributed among stated reasons than the data for sound (Table IV).

We make the following claims based on the results above. We find that students focus on different characteristics of sound, and that these associations are strongly coupled to their choice of representation. On the other hand, while students focus on different characteristics of oscillating strings, a single representation is associated with multiple characteristics, unlike the case for sound. ${ }^{32}$ These results were similar for both semesters.

The results on the motion question were similar in both initial and follow-up studies. Figure 6 shows the fractions of students choosing a particular representation that selected a particular answer to the motion question. For example, in the spring semester, of the students who chose the "sine wave" representation of sound, $43 \%$ answered up and down. The

TABLE III. Long answer coding for sound.

\begin{tabular}{ll}
\hline \hline Category & \multicolumn{1}{c}{ Sample statement } \\
\hline Formalism & "This makes sense to me because when I think of \\
& waves I think of sinusoidal waves." \\
"It's a compression wave that is moving air & particles." \\
"I think that sound waves spread out from a & $\begin{array}{l}\text { source, such that you can hear them in } \\
\text { any position in front of the speaker." }\end{array}$ \\
\hline \hline
\end{tabular}


TABLE IV. Long answer reasons for sound.

\begin{tabular}{lccc|ccc}
\hline \hline & \multicolumn{3}{c}{ Spring } & \multicolumn{3}{c}{ Fall } \\
\cline { 2 - 7 } & Formalism & Medium & Spreads & Formalism & Medium & Spreads \\
\hline Sine & 14 & 1 & 0 & 23 & 0 & 0 \\
Particle & 1 & 15 & 0 & 1 & 10 & 1 \\
Circles & 6 & 18 & 39 & 7 & 12 & 71 \\
\hline \hline
\end{tabular}

“*” indicates the correct answer for each group. In the string group, the majority of students $(>83 \%)$ chose the correct answer (up and down). This choice was independent of their choice of representation $\left(\chi^{2} ; p>0.3\right)$. In contrast, the responses in the sound group were varied. We found a relationship between students' choice of representation and response to the follow-up question $\left(\chi^{2} ; p<0.01\right)$. Students who chose the "sine wave" representation were mostly likely to choose vertical motion (up and down) compared to students choosing other representations, and were least likely to choose the correct answer (side-to-side). Students who chose the "particles" representation (middle) were most likely to choose the correct answer, and students who chose the "circles" representation were most likely to choose "to the right." Thus, we find that for sound, students' choice of representation is connected to a particular answer, while for strings there is no such connection between representation and answer.

\section{DISCUSSION}

We have shown that analogies are generative for a large college physics class, and further demonstrated that analogies can be generative when taught. We use findings on students' use of representations to begin to understand how and why analogies may be taught. Results of the representation assessment demonstrate that different representations may cue students to focus on different characteristics of waves, but only in certain cases were particular representations associated with students' reasoning about the phenomenon. For instance, while particular representations of a wave on a string seem to cue different characteristics, the vast majority of students answered the motion question correctly, and there was no correlation between the answer and choice of representation. Thus, students demonstrated a correct understanding of the phenomenon regardless of representation. Although the representations of strings cue different characteristics, they are not vastly different-all three are variations on the sinusoid. This result points to an important characteristic of a wave on a string, namely that it is a concrete phenomenon. By concrete, we mean that students have direct access to waves on a string via visual input channelsstudents have seen a string moving up and down. Thus, a model of a wave on a string is based on direct experience, and this model constrains the forms of representation that are appropriate. ${ }^{33}$ Further, we might hypothesize that since a model of a wave on a string is concrete, students may already possess certain phenomenologically grounded knowledge of strings, and are able to project this knowledge over any of the three representations.
Unlike the case for strings, we found that students' reasons for choosing a representation of sound waves, as well as their answer to the motion question, were associated with the choice of representation. Students do not always demonstrate a correct understanding of the phenomenon, and their conceptions of sound are tied to particular representations. For instance, answering "up and down" on the motion question is associated with the sine wave representation, and answering "to the right" is associated with the circles representation. This result suggests that, compared to a wave on a string, sound is a more abstract phenomena. By abstract, we mean that students' experience with sound does not necessarily lead to conceptions that easily map to a scientific model. We use abstract in contrast to concrete, or phenomenologically grounded experience, as described above. Students can hear sound, but they do not see the air moving, and they do not directly experience sound as pressure varying in a sinusoidal fashion. The scientific model of sound is air particles moving as a longitudinal wave, spreading out from a source. However, students may base their reasoning about sound on several models other than the scientific model. ${ }^{34}$ Furthermore, it is well known that students' knowledge can be fractured, consisting of unstable bits and pieces rather than stable, robust mental models. ${ }^{35,36}$ Without a firm understanding of the scientific model, students may turn to the resources at hand, such as representations, to make sense of the phenomenon. This finding suggests caution in the best use of analogy and representation, as it may often be difficult to predict the myriad ways students interpret representations. Recognizing this difficulty, we would like to better understand this finding and to begin to explore mechanisms that explain our observations.

Expert physicists use multiple representations (including verbal, graphical, and gestural) and shift easily between representations. ${ }^{37,38}$ To the expert, all three representations of sound are equivalent in that they all stand for the correct model. The ability to apply such metarepresentational

TABLE V. Long answer coding string.

\begin{tabular}{ll}
\hline \hline Category & \multicolumn{1}{c}{ Sample statement } \\
\hline $\begin{array}{l}\text { Formalism } \\
\text { Transverse }\end{array}$ & $\begin{array}{l}\text { "It is like a sine function which I understand." } \\
\text { and down." }\end{array}$ \\
Traveling & "The wave will travel down the rope." \\
Generic motion & "You can see the whole motion of the string and \\
how it changes."
\end{tabular}


TABLE VI. Long answer reasons for string.

\begin{tabular}{lcccc|cccc}
\hline \hline & \multicolumn{9}{c}{ Spring } & \multicolumn{3}{c}{ Fall } \\
\cline { 2 - 9 } & Formalism & Transverse & Traveling & Generic motion & Formalism & Transverse & Traveling & Generic motion \\
\hline Sine & 8 & 3 & 3 & 8 & 10 & 3 & 2 & 2 \\
Standing & 7 & 13 & 5 & 9 & 16 & 11 & 2 & 13 \\
Traveling & 4 & 4 & 15 & 13 & 16 & 13 & 21 & 9 \\
\hline \hline
\end{tabular}

skills $^{39,40}$ is a defining characteristic of scientists, but the particular interpretations that scientists apply must be learned. ${ }^{41}$ Students, new to the ways physicists think and communicate, appear to draw meanings that vary depending on the representations used. ${ }^{42}$ Some researchers have shown that students can rely on iconic interpretations of graphical representations. ${ }^{43}$ Elby describes this as What-You-See-IsWhat-You-Get (WYSIWYG). ${ }^{44}$ WYSIWYG is one particular form of "read-out" strategy 45,46 and describes reasoning along the lines of $x$ means $x$ (e.g., sinusoid goes up means object goes up). WYSIWYG makes specific predictions for sound. Applied to various iconographic representations of sound, WYSIWYG may lead to different models of sound, some correct and some incorrect. Specifically, if students use representations to frame how they think about phenomena, WYSIWYG predicts that the "particles" and "circles" representations will be most closely aligned with the scientific model (e.g., circles spread means sound spreads). Conversely, WYSIWYG predicts that the "sinusoid" will be aligned with a transverse wave model of sound (e.g., up means $u p$ ), with the wave propagating in a straight line from the source (does not spread). In fact, this result is just what we observe on the representational assessment for sound. Applied to various iconographic representations of strings, WYSIWYG predicts varying models for strings. However, since students' choice of representation did not correlate with their answer to the motion question, this suggests that all three string representations map to a transverse wave model of oscillating strings. Based on these observations, we posit that to students, sound waves are more conceptually abstract (or less concrete) than waves on a string. Thus, we might conclude that for abstract concepts, the WYSIWYG effect is more pronounced.

Some researchers have suggested that one mechanism by which people learn abstract ideas is conceptual blending. ${ }^{18}$ We carry this further and suggest blending as a mechanism by which representations come to stand for scientific models. Suppose we blend the sinusoid with an iconographic representation of an oscillating string. Then the sinusoid can stand for the scientific model of the oscillating string (e.g., 2D, transverse wave). Suppose, instead, that the sinusoid is blended with an iconographic representation showing circular wave fronts. Then the sinusoid can stand for a model of sound that carries with it several characteristics of the scientific model (e.g., 3D, longitudinal wave in air). Notably, the same representation may stand for two or more different models.

Returning to the data on teaching using analogy, we may note that cueing and blending can be used to teach about EM waves. Our results show that this teaching method was effective at generating different inferences about EM waves among students taught with different analogies. For example,
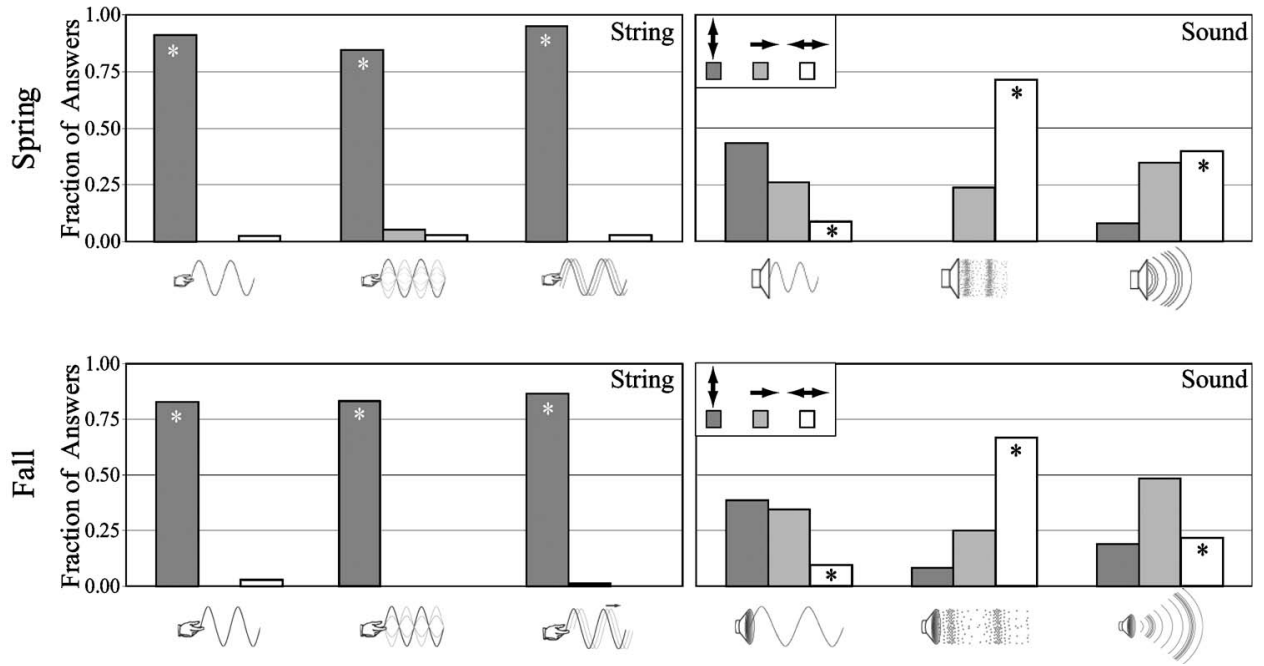

FIG. 6. Student responses to the motion question on the representation assessment. String group (left) and sound group (right). Initial study results (spring) are shown in the top two graphs; follow-up study results (fall) are shown in the bottom graphs. The choice of iconographic representation is shown below the horizontal axis. The three top answers (up/down, to the right, side-to-side) are represented by the directional arrows in the legend. We look for patterns of association between representation and answer. There was no association for string $\left(\chi^{2}: p>0.3\right)$, but significant association for sound $\left(\chi^{2}: p<0.01\right)$. The “*” indicates the correct answer. 
students taught a sound analogy to learn EM predominantly connected the three-dimensional characteristic of sound to EM waves (Fig. 4). Conversely, students that were taught a string analogy were more likely than others to connect the two-dimensional, traveling wave characteristics of waves on a string to EM waves. The different analogies were effective at promoting these connections even though we did not explicitly teach students which mappings to make. We can therefore conclude that the analogies taught different ways of assigning meaning to the same sinusoidal representations. For string, the sine wave stands for a wave confined to the $x-y$ plane, while for sound the sine wave stands for a wave spread throughout space. When this sine wave is used to represent an EM wave, these different characteristics are cued. This cuing explains why choosing the stringlike distracter was associated with the string group, while choosing the soundlike distracter was associated with the sound group.

Noting that both distracters contain elements of the correct answer to the EM wave propagation question (Fig. 3), we might seek to combine the elements present in the "stringlike" distracter (i.e., $1=2=3$ ) with the elements in the "soundlike" distracter (i.e., $1=2=4$ ), in the hopes of achieving the correct answer $(1=2=3=4)$. Thus, we suggest that while either analogy alone is productive for teaching some useful ideas, neither alone is optimized for teaching all of the ideas necessary for a complete understanding of EM waves. It may be that a blend of waves on a string and sound waves will result in a more robust base domain for EM waves. We therefore hypothesize that teaching about EM waves using both analogies may better prepare students to answer the post-test question correctly. Testing this hypothesis directly is the subject of future experimental work. At the same time, understanding why this might be the case suggests the need for an extended framework for analogy. Our results hint that this theoretical framework should include elements of mapping, blending, and read-out strategies such as WYSIWYG. Developing this framework is a subject of future theoretical work.

\section{CONCLUSION}

While previous researchers have demonstrated that analogies generate inferences, they have involved only small numbers of students and demonstrated that teaching with analogies is only sometimes productive. In this study, we found evidence that analogies generate inferences when taught in a large-scale introductory physics course. Furthermore, we have started to delineate a mechanism by which analogies may be taught. We find that representations play a key role as a mechanism of analogy use. Representations appear to cue students to focus on particular characteristics of physical phenomena. Because productive analogy use requires knowledge of which attributes and relations to map between domains, representations and cueing may be used to promote the appropriate use of analogies by students. We caution the reader that these particular studies are designed to understand the role of analogy in student learning, and not necessarily to model the best teaching practices. Nonetheless, these findings lead to the following implications for instruction: (1) Analogies can be used productively to teach in a large enrollment physics course; (2) that when teaching physics with analogies, instructors should attend to the myriad ways representations can be interpreted and may seek to create blends between productive representations. While literal (or WYSIWYG) interpretations may seem naïve to experts, students tend to use such interpretations, and their productive (or unproductive) use depends upon a variety of factors, including instructional environment, framing of analogies, and student resources.

\section{ACKNOWLEDGMENTS}

This work has been supported by the National Science Foundation (Grants No. DUE 0410744 and No. REC CAREER 0448176), the AAPT/AIP/APS (Colorado PhysTEC program), and the University of Colorado. We also wish to extend sincere thanks to Michael Dubson, Murray Holland, Patrick Kohl, and the PER at Colorado Group for their essential and significant contributions to this work.
${ }^{1}$ J. C. Maxwell, in The Scientific Papers of James Clerk Maxwell, edited by W. D. Niven (Cambridge University Press, Cambridge, 1890).

${ }^{2}$ D. Bartlett, Analogies Between Electricity and Gravity, Metrologia 41, S115 (2004).

${ }^{3}$ K. Dunbar, in Model-based Reasoning in Scientific Discovery, edited by L. Magnani, N. Nercessian, and P. Thagard (Plenum Press, New York, 1999).

${ }^{4}$ A. Cho, Ultracold Atoms Spark a Hot Race, Science 301, 750 (2003).

${ }^{5}$ K. Chang, Big Step in Conductivity: More Sociable Particles (New York Times, New York, 2003).

${ }^{6} \mathrm{E}$. Rutherford, The Scattering of $\alpha$ and $\beta$ Particles by Matter and the Structure of the Atom, Philos. Mag. 6, 21 (1911).

${ }^{7}$ D. Gentner, Structure-Mapping: A Theoretical Framework for Analogy, Cogn. Sci. 7, 155 (1983).
${ }^{8}$ D. Gentner and D. R. Gentner, in Mental Models, edited by G. Gentner and A. Stevens (Lawrence Erlbaum Associates, Hillsdale, NJ, 1983).

${ }^{9}$ K. J. Holyoak and P. Thagard, The Analogical Mind, Am. Psychol. 52, 35 (1997).

${ }^{10} \mathrm{G}$. Lakoff, The Contemporary Theory of Metaphor, 2nd ed., in Metaphor and Thought, edited by Ortony and Andrew (Cambridge University Press, Cambridge, 1993).

${ }^{11}$ S. M. Glynn, in The Psychology of Learning Science, edited by S. Glynn, R. Yeany, and B. Beritton (Lawrence Erlbaum Associated, Hillsdale, NJ, 1991).

${ }^{12}$ D. E. Brown and J. Clement, Overcoming Misconceptions via Analogical Reasoning: Abstract Transfer Versus Explanatory Model Construction, Instr. Sci. 18, 237 (1989).

${ }^{13}$ D. E. Brown, Facilitating Conceptual Change Using Analogies and Explanatory Models, Int. J. Sci. Educ. 16, 201 (1994). 
${ }^{14}$ Throughout this paper, we will use the word representation to refer exclusively to external representations (e.g., graphs), rather than internal representation (e.g., mental models).

${ }^{15}$ B. S. Ambrose, P. R. L. Heron, S. Vokos, and L. C. McDermott, Student Understanding of Light as An Electromagnetic Wave: Relating the Formalism to Physical Phenomena, Am. J. Phys. 67, 891 (1999).

${ }^{16}$ Students are also taught that the fields exist in space even if the charges (and, hence, forces) are not present.

${ }^{17}$ D. J. Chalmers, R. M. French, and D. R. Hofstadter, High-level Perception, Representation, and Analogy: A Critique of Artificial Intelligence Methodology, J. Exp. Theor. Artif. Intell. 4, 185 (1992).

${ }^{18} \mathrm{G}$. Fauconnier and M. Turner, The Way We Think: Conceptual Blending and the Mind's Hidden Complexities (Basic Books, New York, 2003).

${ }^{19}$ G. Lakoff and R. Nunez, Where Mathematics Comes From: How the Embodied Mind Brings Mathematics into Being (Basic Books, New York, 2001).

${ }^{20}$ J. Clement, Observed Methods for Generating Analogies in Scientific Problem Solving, Cogn. Sci. 12, 563 (1988).

${ }^{21}$ E. Mazur, Peer Instruction: A User's Manual (Prentice-Hall, New Jersey, 1997).

${ }^{22}$ H-ITT: see http://www.h-itt.com

${ }^{23}$ L. C. McDermott and P. S. Schaffer, Tutorials in Introductory Physics (Prentice-Hall, New Jersey, 2001).

${ }^{24}$ D. Hestenes, M. Wells, and G. Swackhammer, Force Concept Inventory, Phys. Teach. 30, 141 (1992).

${ }^{25}$ L. Bao and E. F. Redish, Model analysis: Representing and assessing the dynamics of student learning, Phys. Rev. ST Phys. Educ. Res. 2, 010103 (2006).

${ }^{26}$ Sound waves are traveling waves, but this was not explicitly taught in the tutorial.

${ }^{27}$ The following analogy may be helpful. During a presidential election, both Democrats and Republicans vote. There are equal numbers in each party, and in both parties more people vote for the Republican. The Republican wins overall, but people who voted for the Democrat were more likely Democrats, while people who voted for the Republican were more likely Republicans.

${ }^{28}$ The two other answers were revised in the follow-up study to be more attractive, but neither proved to be strong distracters.

${ }^{29}$ Post-test results were analyzed using a two-tailed $z$ test of pairwise comparisons.

${ }^{30}$ Since the 3D characteristic was taught explicitly for EM waves in the no-analogy tutorial, students may well have chosen the dis- tracter $1=2=4>3$ more often based on memorization or recall.

${ }^{31}$ Out of 6 in the spring, 8 in the fall.

${ }^{32}$ At this point, we do not know whether representations influence student conceptions of sound, conceptions influence the choice of representation, or if they influence each other.

${ }^{33} \mathrm{We}$ could represent the string with some other representations, such as vectors, but this would not provide any additional important information about the string, nor would it be a typical representation used by an expert.

${ }^{34}$ Z. Hrepic, D. Zollman, and S. Robello, Eliciting and Representing Hybrid Mental Models, Proceedings of the NARST 2005 Annual Meeting (NARST, Dallas, TX, 2005).

${ }^{35}$ A. A. diSessa, in Constructivism in the Computer Age, edited by G. Foreman and P. Pufall, (Lawrence Erlbaum, 1998).

${ }^{36}$ D. Hammer, A. Elby, R. E. Scherr, and E. F. Redish, in Transfer of Learning from a Modern Multidisciplinary Perspective, edited by J. Mestre (Information Age Publishing, Greenwich, CT, 2005), pp. 89-120.

${ }^{37}$ J. Lemke, in Reading Science: Critical and Functional Perspectives on Discourses of Science, edited by J. R. Martin and R. Veels (Routledge, London, 1998).

${ }^{38}$ E. Ochs, P. Gonzales, and S. Jacoby, in Interaction and Grammar, edited by E. Ochs, E. Schegloff, and S. Thompson (Cambridge University Press, Cambridge, 1996).

${ }^{39}$ Metarepresentational competence describes the "full range of capabilities that students (and others) have concerning the construction and use of external representations," (Ref. 40).

${ }^{40}$ A. A. diSessa and B. L. Sherin, Meta-representation: an Introduction, J. Math. Behav. 19, 385 (2000).

${ }^{41}$ W. M. Roth and G. M. Bowen, Complexities of Graphical Representations during Lectures: A Phenomenological Approach, Learn. Instr. 9, 235 (1999).

${ }^{42}$ P. B. Kohl and N. D. Finkelstein, Student Representational Competence and Self-Assessment When Solving Physics Problems, Phys. Rev. ST Phys. Educ. Res. 1, 010104 (2005).

${ }^{43}$ M. Kozhevnikov, M. Hegarty, and R. E. Mayer, Revising the Visualizer-Verbalizer Dimension: Evidence for Two Types of Visualizers, Cogn. Instruct. 20, 47 (2002).

${ }^{44}$ A. Elby, What Students' Learning of Representation tells us about Constructivism, J. Math. Behav. 19, 481 (2000).

${ }^{45}$ A. A. diSessa and B. L. Sherin, What Changes in Conceptual Change? Int. J. Sci. Educ. 20, 1155 (1998).

${ }^{46}$ M. C. Wittman, The Object Coordination Class Applied to Wavepulses: Analysing Student Reasoning in Wave Physics, Int. J. Sci. Educ. 24, 97 (2002). 